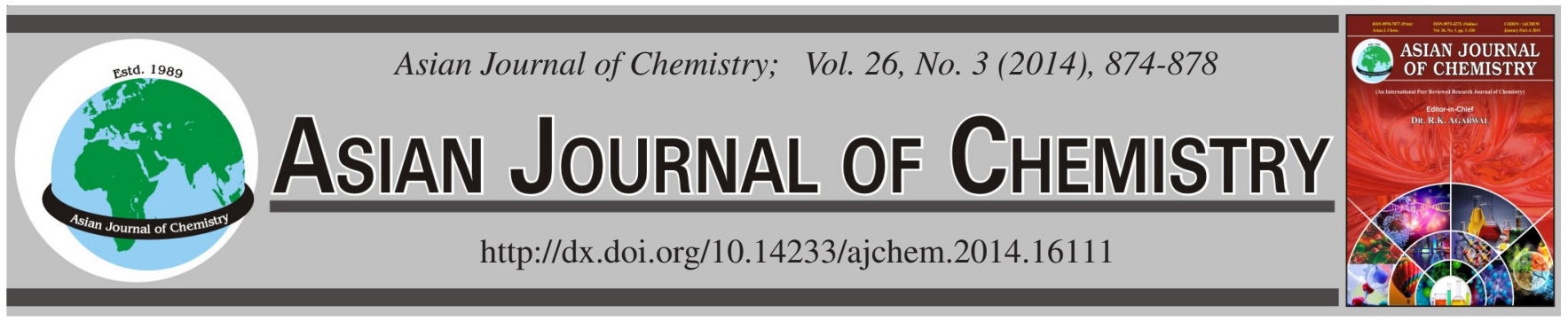

\title{
Quantification of Physiologically Available Glycyrrhizin in Anti-Stress Herbal Formulations by Validated HPTLC Method
}

\section{Nasir A. Siddiqui ${ }^{1, *}$, Perwez Alam ${ }^{1}$, Azmat Ali Khan ${ }^{2}$, Ateeque Ahmad $^{3}$, Adnan J. Alrehaily ${ }^{1}$ and Amer M. Alanazi ${ }^{2}$}

${ }^{1}$ Department Of Pharmacognosy, College of Pharmacy, King Saud University, Riyadh-11451, P.O.Box. 2457, Kingdom of Saudi Arabia ${ }^{2}$ Department Of Pharmaceutical Chemistry, College of Pharmacy, King Saud University, Riyadh-11451, P.O. Box. 2457, Kingdom of Saudi Arabia

${ }^{3}$ Process Chemistry and Product Development Department, CIMAP, Lucknow, India

*Corresponding author: Fax: 00966114677245; Tel: 00966544016921; E-mail: nsiddiqui@ksu.edu.sa

\begin{abstract}
In the present study a simple and novel high-performance thin-layer chromatography (HPTLC) method for quantitative determination of glycyrrhizin at stomach $\mathrm{pH}$ and its comparison with normal $\mathrm{pH}$ has been developed for marketed antistress liquorice root capsules and herbal tea. Chromatography was performed by using solvents including ethyl acetate:glacial acetic acid:methanol:water in proportion of $5: 2: 2: 1, \mathrm{v} / \mathrm{v} / \mathrm{v} / \mathrm{v}$ as mobile phase. The developed plate was scanned and quantified densitometrically at absorption maxima $254 \mathrm{~nm}$. The system was found to give compact spot for glycyrrhizin $\left(R_{f}=0.29 \pm 0.001\right)$. The linearity relationship was described by the equation: $Y$ $=2.103 \mathrm{X}+25.289$. Linearity range for glycyrrhizin was $100-1000 \mathrm{ng}\left(r^{2}=0.996\right)$. The amount of glycyrrhizin was estimated by comparing the peak area of standard and the same was present in the crude extract of antistress herbal formulations. The content of glycyrrhizin in $1 \mathrm{~g}$ of drug material at normal $\mathrm{pH}$ (i.e. 6.8) of methanol was estimated as $7.50 \% \mathrm{w} / \mathrm{w}$ and $6.05 \% \mathrm{w} / \mathrm{w}$ in a dose of sample liquorice root and herbal tea, respectively. At pH 2.58 the glycyrrhizin concentration declined to $5.43 \% \mathrm{w} / \mathrm{w}$ and $3.28 \%$ w/w in sample liquorice root and herbal tea, respectively. The method was validated for precision, accuracy, recovery, robustness, specificity, detection and quantification limits in accordance with ICH guidelines.
\end{abstract}

Keywords: Antistress herbal capsule, High-performance thin-layer chromatography, Glycyrrhizin, Quality control.

\section{INTRODUCTION}

The use of herbs or herbal formulations becomes more challenging because of variability of the constituents. A welldefined and constant composition of the drug is one of the most important prerequisites for the production of a quality herbal formulation. For analytical studies of herbal products various chromatography methods are used among which HPTLC is one of the most useful method ${ }^{1}$. Stress is a very commonly used term in our daily life. The term stress was coined by Hans Selye in $1975^{2}$. The definition of stress may be given as physiological disharmony or threat to homeostasis ${ }^{3}$. Stress usually causes activation of HPA (hypothalamic-pituitaryadrenal) axis through unknown central afferent pathways that stimulate the hypothalamus to release multiple corticotropin (ACTH) secretagogues, corticotropin-releasing hormone $(\mathrm{CRH})$, arginine, vasopressin and the resulting enhanced plasma level of cortisol causes diseases such as hypertension, osteoporosis, depression and the development of the entire spectrum of the metabolic syndrome, including visceral obesity, insulin resistance and dyslipidemia as well as the kinds of cardiovascular diseases ${ }^{4}$. There are many herbal drugs and their formulations like Ocimum sanctum leaves, $R$. damacena root and Panax ginseng roots have been proved their effect as antistress $^{5}$. Some of the herbal drugs are very well known for their adaptogenic, immunomodulatory and antistress properties among which Withania somnifera, Panax ginseng, Asparagus racemosus and Picrorhiza kurroa roots etc. are prominent ${ }^{6-7}$.

Glycyrrhizin, a triterpenoid saponin glycoside from the roots and rhizomes of genus Glycyrrhiza (Licorice, FamilyFabaceae). It is the major bioactive constituent of genus Glycyrrhiza which has been traditionally used in herbal medicine for over 4000 years for the treatment of numerous ailments including liver disorders, stress, allergy, inflammation, spasm, constipation, depression, ulcer, diabetes, dyspepsia, bronchitis, rheumatoid arthritis etc. Licorice is widespread throughout the mediterranean region and certain areas of Asia. Historically, the dried rhizome and root of the plant were used by the Chinese, Egyptian, Greek, Indian and Roman civilizations as expectorant and carminative c-10 $^{8-}$

It is well known that herbal tea (Camellia sinensis) have many medicinal uses due to their major phytoconstituents i.e. 
polyphenols. Herbal teas are used as antioxidants, antimacrobials ${ }^{11}$ and as chemopreventive agents particularly in case of prostate cancer ${ }^{12}$, etc. Now days herbal teas are mixed with some other medicinally useful herbs to modulate their effect according to purpose of use. There are many examples of such drinks like throat clearing herbal teas having herbs like glycyrrhiza, sweet fennel, thyme, etc. ${ }^{13}$ relaxation drink to relieve the stress which generally contain herbs like valerian, kava, etc. that possess tranquilizing, neurocognitive functions and have some role in sleep ${ }^{14}$. Several studies indicate that natural products are susceptible to enzymatic, acid, base hydrolysis and degradation by oxidation and photo oxidation it is therefore necessary to evaluate the extent of degradation particularly in case of formulations meant for oral administration. The hepatoprotective silymarin undergoes acidic and alkaline degradation and it is estimated by HPTLC method ${ }^{15}$. HPTLC method is also used to determine curcumin degradation under stress conditions like acidic, basic, light and oxidation ${ }^{16}$. Glycyrrhizin being a glycoside is also susceptible for hydrolysis at acidic $\mathrm{pH}$ inside stomach.

Nowadays HPTLC is becoming a routine analytical technique due to many advantages like low operating cost; high sample throughput and need for minimum sample clean up, several samples can be run simultaneously using a small quantity of mobile phase unlike HPLC, thus lowering analysis time and cost per analysis ${ }^{17}$. TLC and HPTLC are methods commonly applied for the identification, the assay and the testing for purity, stability, dissolution or content uniformity of raw materials (herbal and animal extracts, fermentation mixtures, drugs and excipients) and formulated products (pharmaceuticals, cosmetics, nutraceuticals $)^{18}$.

\section{EXPERIMENTAL}

The commercial formulations "Licorice root capsules" and "Herbal tea" were purchased from Riyadh, Kingdom of Saudi Arabia. These formulations are frequently used for stress relief and possess glycyrrhizin as major bioactive constituent. liquorice root capsules contains only powdered root of Glycyrrhiza and herbal tea contains flavoring agent, coloring agent and sweeteners along with root powder of Glycyrrhiza plant.

The standard glycyrrhizin was obtained from Sigma Aldrich, Bayouni Trading Co. Ltd. Al-Khobar, Saudi Arabia. The following solvents and regents were used for performing the experiments: ethanol, hexane, ethyl acetate and sulfuric acid. All reagents, chemicals and solvents were of analytical grade, purchased from WINLAB and BDH (U.K.). HPTLC was performed on $10 \times 20 \mathrm{~cm}$ glass-backed silica gel $60 \mathrm{~F} 254$ HPTLC plates from E. Merck (Darmstadt, Germany).

HPTLC instrumentation and conditions: Chromatography was performed, as described by Faiyazuddin et al. ${ }^{17}$, 2010 on $10 \mathrm{~cm} \times 20 \mathrm{~cm}$ glass HPTLC plates precoated with $200 \mu \mathrm{m}$ layers of silica gel 60F254. Samples were applied as bands $6 \mathrm{~mm}$ wide and $8 \mathrm{~mm}$ apart by means of Camag Linomat IV sample applicator equipped with a $25-\mu \mathrm{L}$ syringe. The constant application rate was $160 \mathrm{~nL} \mathrm{~S}^{-1}$. Linear ascending development with solvents EA:GAA: $\mathrm{MeOH}: \mathrm{H}_{2} \mathrm{O}$ (5:2:2:1, $\mathrm{v} / \mathrm{v} / \mathrm{v} / \mathrm{v}$ ) as mobile phase was performed in a $20 \mathrm{~cm} \times 10 \mathrm{~cm}$ twin-trough glass chamber (Camag) previously saturated with mobile phase for $20 \mathrm{~min}$ at room temperature $\left(25 \pm 2{ }^{\circ} \mathrm{C}\right)$ and relative humidity $60 \pm 5 \%$. The development distance was $7.2 \mathrm{~cm}$ (development time $10 \mathrm{~min}$ ) and $10 \mathrm{~mL}$ mobile phase was used. The plates were dried at room temperature and then heated to identify compact bands. Densitometric analysis was performed at $254 \mathrm{~nm}$ in absorbance/reflectance mode with a Camag TLC scanner III operated by WinCATS 4 software (Version 1.2.0). The slit dimensions were $5 \mathrm{~mm} \times 0.45 \mathrm{~mm}$ and the scanning speed of $20 \mathrm{~mm} \mathrm{~S}^{-1}$.

Extraction conditions: The protocol for preparing sample solutions was optimized for high quality fingerprinting and also to extract the marker compound efficiently. Since the marker compound was soluble in methanol, therefore methanol was used for extraction. The fingerprinting of methanol extracts of both the samples was executed by spotting with suitably diluted sample solution on a HPTLC plate. Each amount was applied in triplicate. Peak area and amounts applied were treated by linear least squares regression. The plates were developed and scanned at $254 \mathrm{~nm}$ in absorbance/ reflectance mode with a Camag TLC scanner III operated by WinCATS 4 software (Version 1.2). The peak areas were recorded and the amount of glycyrrhizin was calculated using the calibration curve.

Preparation of sample from Licorice root capsules: C1: Two capsules of sample weighing $1000 \mathrm{mg}$ powdered drug material have been extracted with $10 \mathrm{~mL}$ methanol by ultrasonic method for $1 \mathrm{~h}$ and then filtered by vacuum filtration. The same procedure was repeated again with marc to exhaust the drug material. Two filtrates were combined and concentrated to $10 \mathrm{~mL}$. From this stock solution $1 \mathrm{~mL}$ was taken and diluted to $10 \mathrm{~mL}$ with the help of methanol.

C2: The procedure similar to $\mathrm{C} 1$ was followed and $\mathrm{pH}$ 2.58 was adjusted with the help of concentrated hydrochloric acid.

Preparation of sample from herbal tea: P1: Herbal tea weighing $1000 \mathrm{mg}$ has been extracted with $10 \mathrm{~mL}$ methanol by ultrasonic method for $1 \mathrm{~h}$ and then filtered by vacuum filtration. The same procedure was repeated again with marc to exhaust the drug material. Two filtrates were combined and concentrated to $10 \mathrm{~mL}$. From this stock solution $1 \mathrm{~mL}$ was taken and diluted to $10 \mathrm{~mL}$ with the help of methanol.

P2: The procedure similar to $\mathrm{P} 1$ was followed and $\mathrm{pH}$ 2.58 was adjusted with the help of concentrated hydrochloric acid (Conc. $\mathrm{HCl})$.

Preparation of sample from standard: Stock solution of glycyrrhizin standard $\left(1 \mathrm{mg} \mathrm{mL}^{-1}\right)$ was prepared in methanol and by appropriate dilution it was made to the concentration of $0.1 \mu \mathrm{g} / \mu \mathrm{L}$. For calibration, glycyrrhizin standard solution (1-10 $\mu \mathrm{L})$ was applied to a HPTLC plate to furnish amounts in the range $100-1000 \mathrm{ng}$ band $^{-1}$.

Methanol solution $(0.1 \mathrm{mg} / \mathrm{mL})$ of standard and formulations extract $(5 \mu \mathrm{L})$ were applied to chromatographic plates bandwise, by means of a camag automatic TLC sampler- IV and developed in ADC2 (automatic development chamber). Plates, after derivatization, were documented with the use of Camag TLC Reprostar 3 with computer program Videostore and scanned with densitometer Camag TLC scanner with computer program CATS 4 (Camag). 


\section{RESULTS AND DISCUSSION}

The amount of glycyrrhizin was determined from the calibration curve (Fig. 1) obtained by plotting the concentration of glycyrrhizin standard against the peak area. The content of glycyrrhizin was estimated in the methanol extract samples of liquorice root capsules and herbal tea at normal $\mathrm{pH}$ by the proposed method was $7.50 \% \mathrm{w} / \mathrm{w}$ and $6.05 \% \mathrm{w} / \mathrm{w}$ of powdered drug material respectively. At $\mathrm{pH} 2.58$ the concentration of glycyrrhizin goes down to $5.43 \% \mathrm{w} / \mathrm{w}$ and $3.28 \% \mathrm{w} / \mathrm{w}$ of powdered drug material of liquorice root capsules and herbal tea respectively. The percentage degradation in herbal tea was found to be more $(39.48 \%$ ) than liquorice root capsules $(19.38 \%)$. The difference in the percent degradation of liquorice root capsules and herbal tea may be due to the presence of certain other constituents like coloring agents, flavoring agents, sweeteners etc. which were present in herbal tea while liquorice root capsules contains only root powder of glycyrrhiza. Hydrolytic effect of conc. $\mathrm{HCl}$ is clearly demonstrated in Figs. 3 and 4 where the difference in peak area of both the samples at two different $\mathrm{pH}$ can be observed. Again the selected peak of glycyrrhizin in both the samples at normal $\mathrm{pH}$ and at $\mathrm{pH}$ 2.58 shows marked difference in the peak area. Therefore this experiment proves that after oral ingestion the estimated quantity of glycyrrhizin will be the physiologically available quantity to the human body. After getting the degradation results of marker compound the quantity of powdered drug to be filled in capsules or the quantity of herbal tea to be taken as antistress can be easily be decided. It is for the first time, a simple, accurate and rapid HPTLC method has been developed for the quantification of physiologically available bioactive marker glycyrrhizin in different herbal formulations of Glycyrrhiza mainly prescribed as antistress agent.

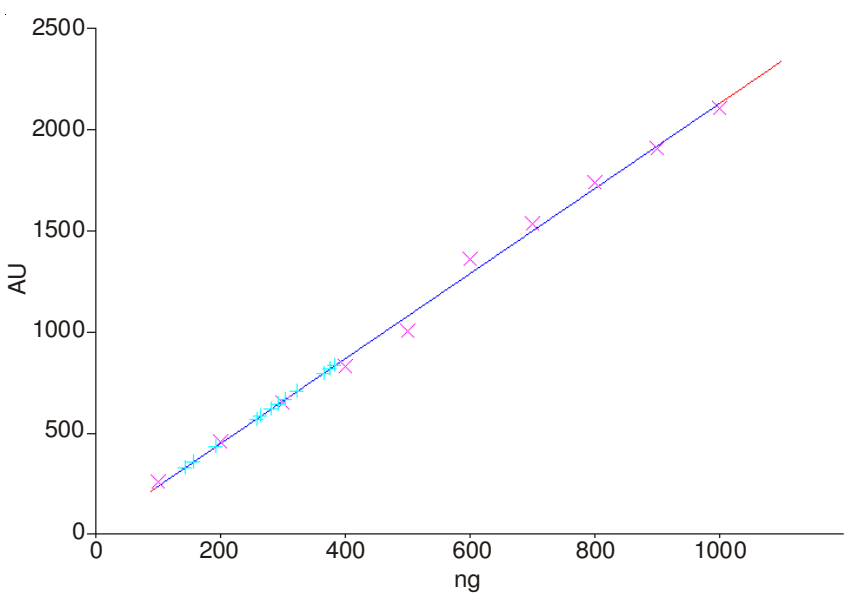

Fig. 1. Calibration curve of standard glycyrrhizin@ @254 nm

Chromatography: Chromatogram was developed for glycyrrhizin standard under chamber saturation conditions using solvents Ethyl acetate: GAA: $\mathrm{MeOH}: \mathrm{H}_{2} \mathrm{O}$ (5:2:2:1, $\mathrm{v} / \mathrm{v} / \mathrm{v} / \mathrm{v}$ ) as mobile phase (Fig. 2). The same mobile phase has been also employed for the separation of components in methanolic extracts of sample liquorice root capsules and herbal tea (Figs. 3 and 4, respectively). The optimized saturation time was found to be $10 \mathrm{~min}$. UV spectra measured for the
TABLE-1

$\mathrm{R}_{f}$, LINEAR REGRESSION DATA FOR THE CALIBRATION CURVE AND SENSITIVITY PARAMETER FOR GLYCYRRHIZIN

\begin{tabular}{ll}
\hline \multicolumn{1}{c}{ Parameter } & \multicolumn{1}{c}{ Glycyrrhizin } \\
\hline \multicolumn{1}{c}{$\mathrm{R}_{\mathrm{f}}$} & 0.29 \\
Linearity range (ng band ${ }^{-1}$ ) & $100-1000$ \\
& \\
Regression equation & $\mathrm{Y}=2.103 \mathrm{X}+25.289$ \\
Correlation coefficient & $\mathrm{r}^{2}=0.996$ \\
Slope $\pm \mathrm{sd}$ & $2.103 \pm 0.003$ \\
Intercept \pm sd & $25.289 \pm 0.005$ \\
Standard error of slope & 0.001 \\
Standard error of intercept & 0.001 \\
LOD & $34 \mathrm{ng}$ \\
LOQ & $101 \mathrm{ng}$ \\
\hline
\end{tabular}

spots showed maximum absorbance at $254 \mathrm{~nm}$ in the absorbance/ reflectance mode. Compact bands as sharp, symmetrical and with high resolution were obtained at $R_{f} 0.29 \pm 0.001$.

Calibration curve: Linearity of compound glycyrrhizin was validated by the linear regression equation and correlation

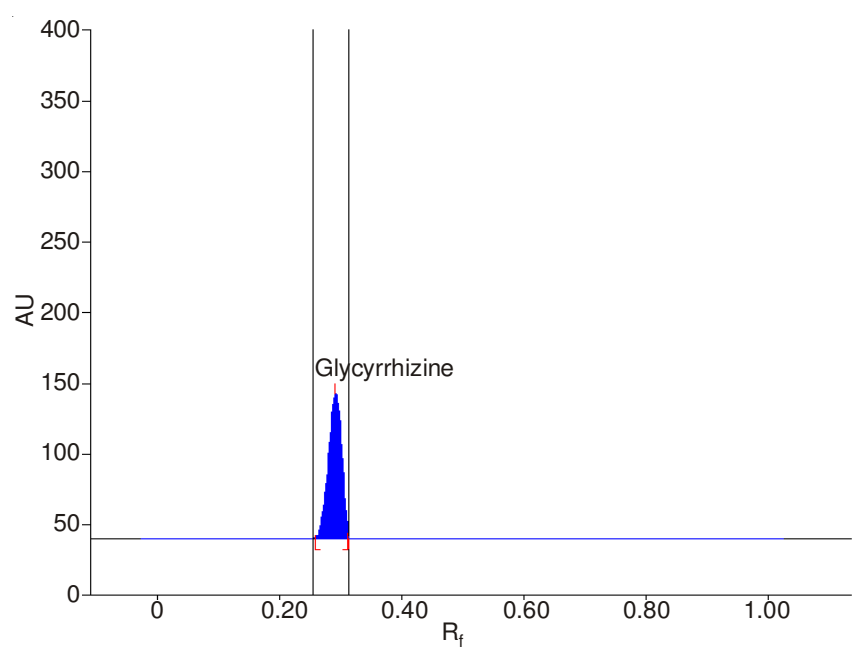

Fig. 2 HPTLC chromatogram of standard glycyrrhizin with mobile phase ethyl acetate : galacial acetic acid : methanol : water in proportion of $5: 2: 2: 1, \mathrm{v} / \mathrm{v} / \mathrm{v} / \mathrm{v}$ scanned at $254 \mathrm{~nm}$

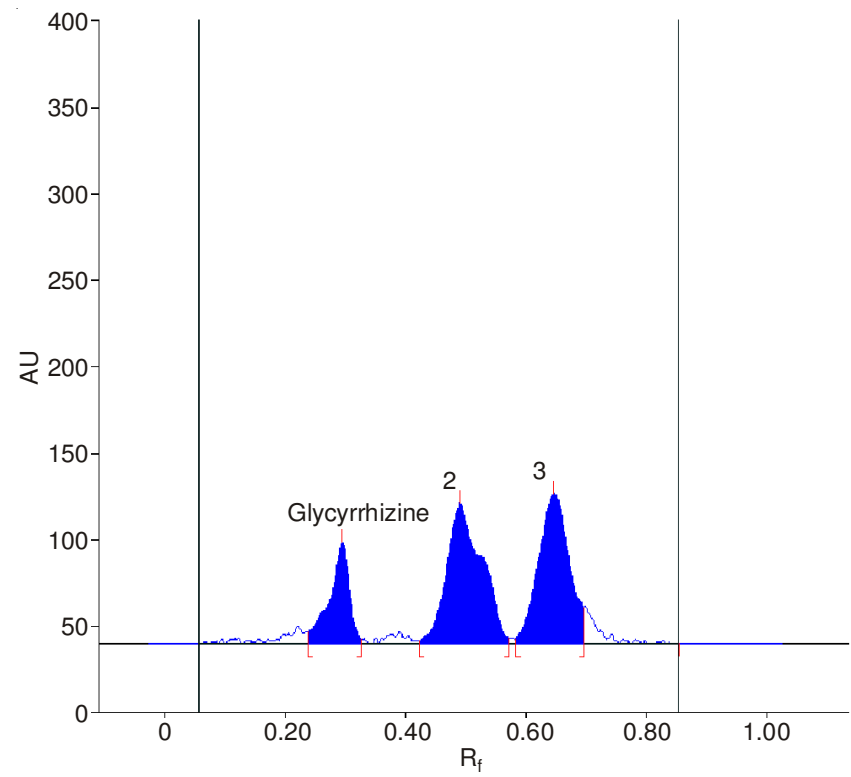

(i) 


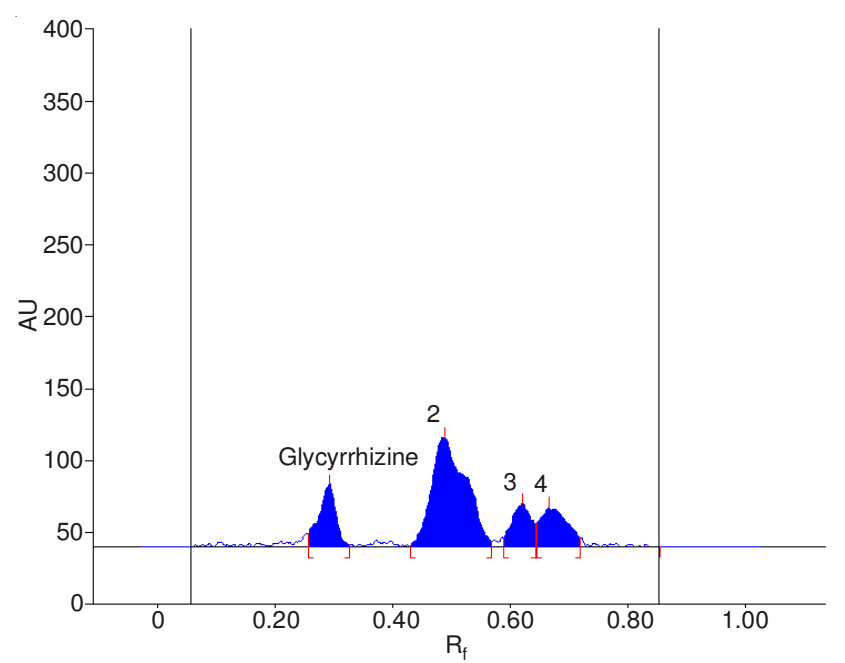

(ii)

Fig. 3. (i) Components of liquorice root capsules at normal pH (ii): Components of liquorice root capsules at $\mathrm{pH} 2.58$

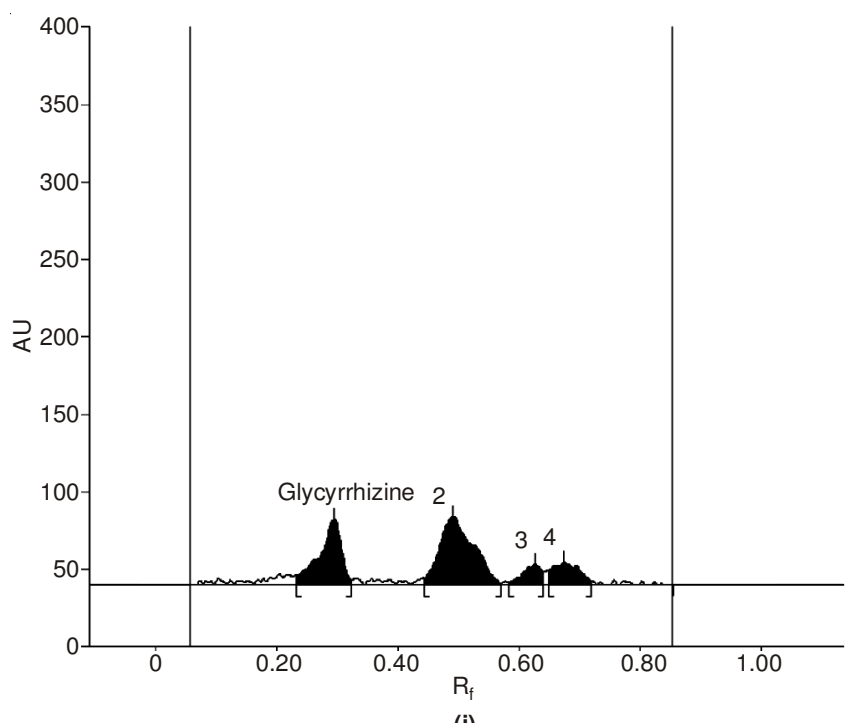

(i)

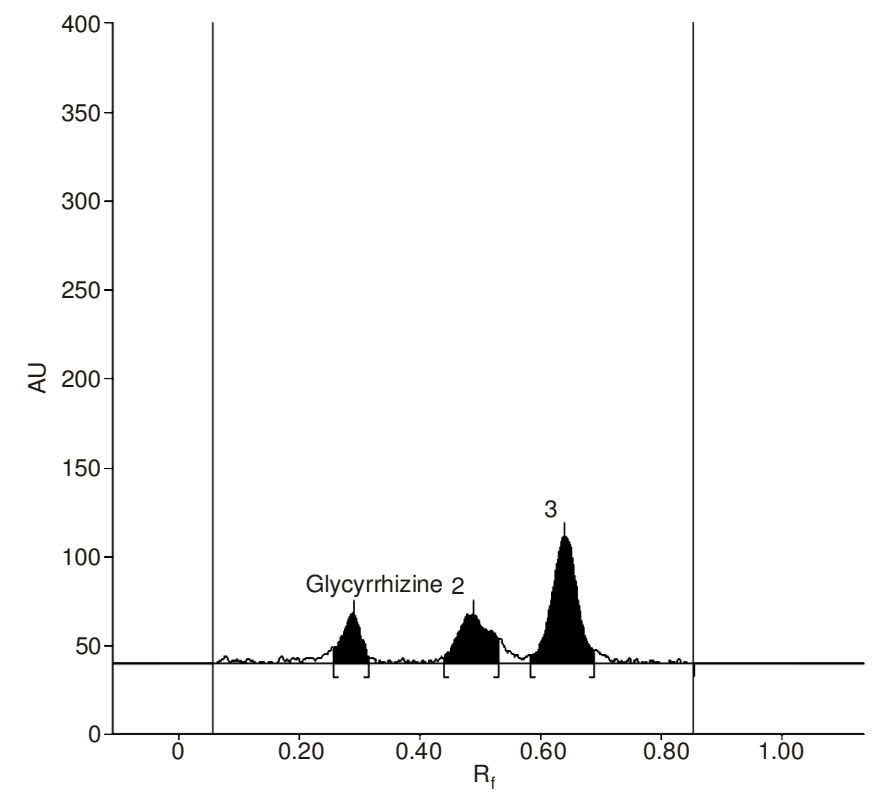

(ii)

Fig. 4. (i) Components of herbal tea at normal pH (ii) Components of herbal tea at $\mathrm{pH} 2.58$ coefficient. The ten-point calibration curve (Fig. 1) for glycyrrhizin was found to be linear in the range of 100-1000 ng. Regression equation and correlation coefficient for the reference compound was $\mathrm{Y}=2.103 \mathrm{X}+25.289\left(\mathrm{r}^{2}=0.996\right)$ which revealed a good linearity response for developed method and are presented in Table-1.

Glycyrrhizin was well resolved at $\mathrm{R}_{\mathrm{f}} 0.29$ from $\mathrm{MeOH}$ extracts of liquorice root capsules and herbal tea in the solvent system as mentioned above. The plate was visualized at wavelength $254 \mathrm{~nm}$ as the compound was found to absorb at this maxima. The method developed here was found to be quite selective with good baseline resolution of each compound. The identity of the bands of compounds in the sample extracts were confirmed by overlaying their absorption spectra with those of the standards at $254 \mathrm{~nm}$ (Fig. 5).

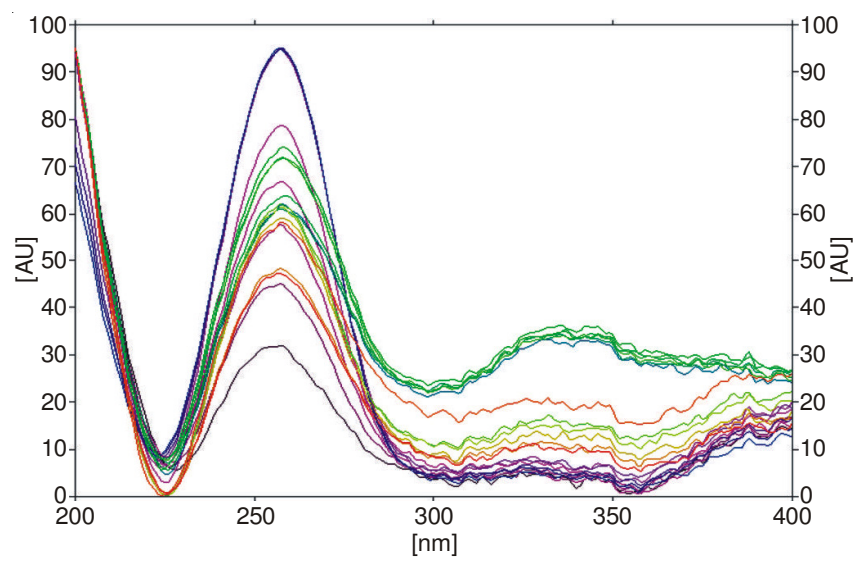

Fig. 5. Spectral comparison of glycyrrhizin with $\mathrm{MeOH}$ extracts of sample liquorice root capsules and herbal tea with mobile phase ethylacetate: GAA: $\mathrm{MeOH}: \mathrm{H}_{2} \mathrm{O}(5: 2: 2: 1$, v/v/v/v)

Method validation: The method adopted here was validated for precision, accuracy, robustness, sensitivity and recovery studies. Intra-day precisions $(\mathrm{n}=6)$ for glycyrrhizin was found to be $=1.84 \%$, however the inter-day precisions was $=1.62 \%$, which demonstrated the good precision of proposed method. Intra-day accuracy of glycyrrhizin was 99.2 - $99.7 \%$, however interday accuracy for glycyrrhizin was 98.9-99.6\%. These values are within the acceptable range, so the method was accurate, reliable and reproducible. The SD and \% RSD was calculated for glycyrrhizin. The low value of SD and \% RSD obtained after introducing small deliberate changes in the method indicated that the method was robust. LOD and LOQ values for glycyrrhizin were found to be 34 and $101 \mathrm{ng} \mathrm{band}^{-1}$, respectively, indicating adequate assay sensitivity. The LOD and LOQ were determined from the slope of the lowest part of the calibration plot. This indicated that the proposed method exhibits a good sensitivity for the quantification of above compounds. Good recoveries were obtained by the fortification of the sample at three quality control levels for glycyrrhizin. It is evident from the results that the percent recoveries for glycyrrhizin after sample processing and applying were in the range of $99.4-99.9 \%$.

\section{Conclusion}

A validated HPTLC method has been developed for the determination of Glycyrrhizin at normal as well as acidic $\mathrm{pH}$ 
in different herbal formulations of Glycyrrhiza (liquorice root capsules and herbal tea) marketed in Kingdom of Saudi Arabia as stress reliever. The present study has clearly given an evidence of $\mathrm{pH}$ related degradation of the active constituents. As far as quality control of herbals are concern the formulations must be analyzed for their possible ways of degradations which may include exposure to high temperature, moisture, acids, alkali, enzymes, oxidation and photo-oxidation conditions etc.

This approach of evaluation can give an approximation about the quantity of active ingredients available physiologically to the body. Statistical analysis proved that the method is authentic and reproducible for the analysis of glycyrrhizin. To make sure the presence of therapeutic dose of active constituents in herbal formulations, HPTLC method is very useful for quantification of marker compounds in the raw material as well as in finished products. This is one of the reliable and economic methods to produce quality herbal formulations.

\section{ACKNOWLEDGEMENTS}

The authors extended their sincere appreciation to the Deanship of Scientific Research at King Saud University for its funding of this research through the Research Group Project No. RGP-VPP-212.

\section{REFERENCES}

1. O.F. Kunle, H.O. Egharevba and P.O. Ahmadu, Int. J. Biodivers. Conserv., 4, 101 (2012).
2. H. Selye, J. Human Stress, 1, 37 (1975).

3. M. Sumanth and S.S. Mustafa, Int. J. Pharmacol, 3, 416 (2007).

4. R. Rosmond, Psychoneuroendocrinology, 30, 1 (2005).

5. V. Singh, A. Singh, R. Nath, N. Mishra, K.S. Dixit and N. Singh, J. Biol. Chem. Res, 10, 601 (1991).

6. D.C. Anderson, Integr. Med., 7, 18 (2008).

7. N.A. Siddiqi, S. Singh, M.M. Siddiqui and T.H. Khan, Int. J. Pharmacol,, 8, $108(2012)$

8. Q. Afnan, M.D. Adil, A. Nissar-Ul, A.R. Rafiq, H.F. Amir, P. Kaiser, V.K. Gupta, R. Vishwakarma and S.A. Tasduq, Phytomedicine, 19, 658 (2012).

9. C.V. Chandrasekaran, K. Sundarajan, A. Gupta, H.S. Srikanth, J. Edwin and A. Agarwal, Regul. Toxicol. Pharmacol., 61, 373 (2011).

10. E.W.C. Chan, P.P. Tie, E.Y. Soh and Y.P. Law, Pharmacog. Res., 3, 266 (2011).

11. J.J. Johnson, H.H. Bailey and H. Mukhtar, Phytomedicine, 17, 3 (2010).

12. G. Puodziuniene, V. Janulis, A. Milasius and M. Budnikas, Medicina (Kaunas), 40, 762 (2004).

13. S. Stacy, J. Child Adolesc. Psychopharmacol., 21, 605 (2011).

14. Y.J. Li, J. Chen, Y. Li, Q. Li, Y.F. Zheng, Y. Fu and P. Li, J. Chromatogr. A, 1218, 8181 (2011).

15. R. Parveen, S. Ahmad, S. Baboota, J. Ali and A. Alka, Biomed. Chromatogr., 24, 639 (2010).

16. M.J. Ansari, S. Ahmad, K. Kohli, J. Ali and R.K. Khar, J. Pharm. Biomed. Anal., 39, 132 (2005).

17. M. Faiyazuddin, J. Ali, S. Ahmad, N. Ahmad, J. Akhtar and S. Baboota, J. Planar Chromatogr., 23, 233 (2010).

18. G. Biringanine, M.T. Chiarelli, M. Faes and P. Duez, Talanta, 69, 418 (2006). 